\title{
An Electrophysiological Investigation of Factors Facilitating Strategic Recollection
}

\author{
J. E. Herron and E. L. Wilding
}

\begin{abstract}
Episodic memory is thought to be mediated by executive processes that facilitate the retrieval of task-relevant information at the expense of irrelevant information. The exclusion task [A process dissociation framework: Separating automatic from intentional uses of memory. Journal of Memory and Language, 30, 513-541, 1991] can be used to explore these processes. In this task, studied items from one source ("targets") are endorsed on one response key, whereas new and studied items from another source ("nontargets") are rejected on another key. Herron and Rugg [Strategic influences on recollection in the exclusion task: Electrophysiological evidence. Psychonomic Bulletin and Review, 10, 703-710, 2003] reported that nontargets elicited the ERP correlate of recollection (the "left parietal old/new effect") when target accuracy was low, but not when it was high. Their explanation
\end{abstract}

for this was that participants only focused exclusively on the recollection of target information when the likelihood of target recollection was high, as under these conditions this strategy is one that that will give rise to accurate task performance. The fact, however, that targets were encoded in different tasks in the high- and low-accuracy groups means that the results can also be explained in terms of the encoding operations performed at study rather than in terms of target accuracy. This study was designed to distinguish between these competing accounts. All targets were encoded elaboratively. Target accuracy was reduced in one condition with a 40-min study-test interval. Nontargets elicited no left parietal effect in either condition, suggesting that target-specific strategic retrieval is facilitated by certain classes of encoding operations rather than simply high target accuracy per se.

\section{INTRODUCTION}

It is generally held that episodic memory is mediated by control processes that guide retrieval in order to recover selectively contextual information relevant to the task at hand (a concept henceforth referred to as "strategic retrieval"). Such control processes are arguably essential in order to prevent the rememberer from being inundated with task-irrelevant information. In recent years, ERP experiments employing the exclusion task (Jacoby, 1991) have revealed findings pertinent to strategic retrieval. The exclusion task was introduced as a component of Jacoby's (1991, 1998) process dissociation procedure, a method of providing behavioral estimates of the relative contributions of familiarity and recollection to recognition memory performance. In a typical exclusion task, participants are presented with two different study phases/contexts and are then required to respond positively to items from one phase/context at test (targets) and to reject items from the alternate phase or context (nontargets) together with new items. Although Jacoby proposed that nontargets are excluded successfully only when participants recollect contextual information that is diagnostic of the item's source, it is

Cardiff University

also possible to complete this task without recollecting any nontarget information, as nontargets will also be classified correctly if they are forgotten. The question therefore arises: If participants adopt a target-specific retrieval strategy (i.e., attempt to recollect target information only), will nontargets be recollected?

The fact that new items and nontargets share the same response means that it is not possible to determine the basis for a correct nontarget judgment (whether nontarget information has been recollected) on the basis of behavioral data alone. ERPs are a useful tool in addressing this issue because a wealth of evidence from a variety of ERP studies has converged to identify a neural correlate of recollection, the left parietal old/ new effect, which takes the form of greater positivity for correctly identified old items than for correctly identified new items between 500 and $800 \mathrm{msec}$, a positivity that is maximal in amplitude at left parietal scalp sites (see Friedman \& Johnson, 2000; Rugg \& Allan, 2000, for reviews). The amplitude of the left parietal effect increases with the amount of contextual information retrieved (Wilding, 2000) and is also larger for recognized items attracting "remember" responses than "know" responses (Duzel, Yonelinas, Mangun, Heinze, \& Tulving, 1997; Smith, 1993, although see Spencer, Abad, \& Donchin, 2000). 
The combined use of ERPs together with the exclusion task can therefore be valuable in addressing the issue of strategic retrieval. The critical question is whether the left parietal old/new effect is observed for correctly classified nontargets. Although certain studies have reported significant nontarget left parietal effects in exclusion tasks that employ target/nontarget distinction criteria such as voice (Wilding \& Sharpe, 2004; Wilding \& Rugg, 1997) and color (Cycowicz, Friedman, \& Snodgrass, 2001), several recent studies have found that correctly classified nontargets do not always elicit a nontarget left parietal effect (Herron \& Rugg, 2003a, 2003b; Dywan, Segalowitz, \& Webster, 1998), indicating that these items are not recollected under certain experimental conditions. Dywan et al. (1998) employed an exclusion task in which participants were required to identify studied items during a memory test incorporating studied items, new items, and repeating lures (all items were visually presented words). Whereas studied items (targets) elicited a significant left parietal old/new effect, no such effect was detected for the nontarget repeating lures (see Dywan, Segalowitz, \& Arsenault, 2002; Dywan, Segalowitz, Webster, Hendry, \& Harding, 2001, for replications). More recently, Herron and Rugg (2003b) presented targets and nontargets together in a single study phase, the two classes of study items being differentiated from each other by their symbolic form at study; one class was presented as pictures, whereas the other class was visually presented words (participants were also instructed to complete different elaborative encoding tasks according to the symbolic form of the stimulus). Items studied as pictures were designated as targets in one condition, whereas items studied as words were targets in the other. All test stimuli were words. A nontarget left parietal effect was evident when pictures were targets, but not when words were targets. It was argued that the high level of cue-target compatibility when words were designated as targets allowed participants to search memory with a higher degree of specificity than when pictures were targets, and that nontarget information was consequently not retrieved in this condition. These findings suggest that the retrieval of nontarget information can be suppressed or inhibited under certain conditions.

In a further study, Herron and Rugg (2003a) employed a between-participants design in which target memorability was manipulated through the use of encoding task (all study and test items were visually presented words); Group 1 encoded targets in a "shallow" task (read aloud) and Group 2 encoded targets in a "deep" task (pleasantness rating). All nontargets were encoded in the same elaborative task (sentence generation). Participants were required to respond positively to targets at test and to exclude both nontargets and new items. Nontargets elicited a left parietal effect when target accuracy was relatively low (0.63) but not when target accuracy was high (0.76). The authors proposed that when memory for targets was good, participants focused exclusively on the retrieval of target information at the expense of nontarget information, and that when target memory was insufficiently reliable to support such a strategy, participants attempted to retrieve both target and nontarget information. It was concluded that levels of target accuracy may influence what retrieval strategy participants adopt in the exclusion task, and that the retrieval of nontarget information may consequently be influenced directly by target accuracy.

As levels of target accuracy were confounded with target-encoding task, however, an alternative hypothesis arises; it is possible that target-specific retrieval strategies are also influenced by the nature of the encoding operations performed at study and not just by target accuracy. For example, participants may only be able to selectively retrieve targets that have been manipulated in a sufficiently elaborative encoding task or in a task that is sufficiently distinct from that performed on nontargets. The present study was designed to test this hypothesis by varying target accuracy, but without confounding accuracy with encoding task. Instead, all targets were encoded using the same pleasantness-rating task used by Herron and Rugg (2003a; deep condition), but target accuracy was lowered in one condition by inserting a 40-min interval after the target study phase. In both conditions, the nontarget encoding task was the same (animacy judgments). If the hypothesis that target accuracy influences directly the retrieval of nontarget information is correct (i.e., that nontarget information is recollected whenever target accuracy is low), then a left parietal effect should still be observed for nontargets in the present experiment when target accuracy is low.

\section{RESULTS}

All analyses included the Greenhouse-Geisser correction for nonsphericity where necessary, and epsiloncorrected degrees of freedom are given in the text (Greenhouse \& Geisser, 1959). An alpha level of .05 was used for all statistical tests. The term "difficulty" will be used to refer to the interval manipulation; "difficult" refers to the experimental block in which a 40-min interval separated the target and nontarget study tasks. The block in which no interval was employed is referred to as the "easy" condition. The term "response category" will be used to refer to the three types of correct response; the nomenclature "target," "nontarget," and "new" will be adopted for correct responses to targets, nontargets, and new items, respectively.

\section{Behavioral Data}

Accuracy and reaction time (RT) data are displayed in Table 1. The likelihood of a target judgment to a target 
Table 1. Response Accuracy and RTs for Targets, Nontargets, and New Items in the Difficult and Easy Conditions

\begin{tabular}{lcr}
\hline & Proportion & $R T$ \\
\hline Difficult & $.65(.15)$ & $1086(183)$ \\
Target & $.83(.10)$ & $1121(157)$ \\
Nontarget & $.96(.04)$ & $943(133)$ \\
New & & \\
Easy & $.72(.15)$ & $1053(152)$ \\
Target & $.82(.09)$ & $1092(226)$ \\
Nontarget & $.93(.08)$ & $921(181)$ \\
New &
\end{tabular}

The table shows the probabilities of correct responses to each item type, as well as the averaged RTs associated with each type of correct response (standard deviations in parentheses).

was reliably greater than the likelihood of a target judgment to a nontarget or a new word in both conditions, $t(17)>18, p<.001$, in each case. ANOVA of the accuracy data incorporating the factors of Difficulty (easy/difficult) and Response Category (correct target/ nontarget/new) gave rise to a main effect of Response Category, $F(1.3,21.9)=36.71, p<.001$, and a Difficulty $\times$ Response Category interaction, $F(1.6,26.6)=$ $5.55, p<.025$. Pairwise comparisons indicated that target accuracy was significantly lower in the difficult condition than in the easy condition, $F(1,17)=6.76, p<.025$, whereas Difficulty did not influence either nontarget or new item accuracy. ANOVA of the corresponding RT data gave rise to no significant effects involving either Response Category or Difficulty.

\section{ERPs}

The mean numbers of trials (range in parentheses) contributing to the averaged ERPs elicited by targets, nontargets, and new items were 42 (25-61), 58 (24-67), and $63(30-76)$ in the difficult condition, and $46(16-66)$, 55 (37-70), and 60 (36-76) in the easy condition. Averaged ERPs from left and right parietal electrodes are shown in Figure 1A. In both conditions, target ERPs show an increased positivity relative to new and nontarget ERPs at the left parietal site between 500 and $800 \mathrm{msec}$. This positivity is also evident at mid and right frontal sites during the same latency region, although the positivity at these sites is sustained until the end of the recording epoch (see Figure 2). This "right frontal old/new effect" has been reported previously (e.g., Rugg, Allan, \& Birch, 2000; Ullsperger, Mecklinger, \& Muller, 2000; Donaldson \& Rugg, 1998; Wilding \& Rugg, 1996) and may reflect aspects of postretrieval processing such as the monitoring and/or evaluation of retrieved information (see Rugg \& Allan, 2000, for a review). An additional old/new effect of reversed polarity is evident at $\mathrm{Pz}$ for targets and nontargets in both conditions (see Figure 2). This effect-the "late posterior negativity"-may reflect a combination of responselocked and stimulus-locked retrieval-related processes (Johannsen \& Mecklinger, 2003). Finally, also evident in Figure $1 \mathrm{~B}$ is an early positivity for both targets and nontargets relative to new items, maximal at frontal sites between 300 and $500 \mathrm{msec}$. According to recent accounts, this old/new effect is an index of familiarity (Rugg, Herron, \& Morcom, 2002; Curran, Schacter, Johnson, \& Spinks, 2001; Curran, 2000; Rugg et al., 1998). This early midfrontal effect appears to be larger in the easy condition.

All four old/new effects were explored in a set of a priori focused analyses that were guided by the literature pertaining to each old/new effect. All analyses incorporated the factors of Difficulty (easy/difficult) and Response Category (targets, nontargets, and new items). Only significant effects involving Response Cate-

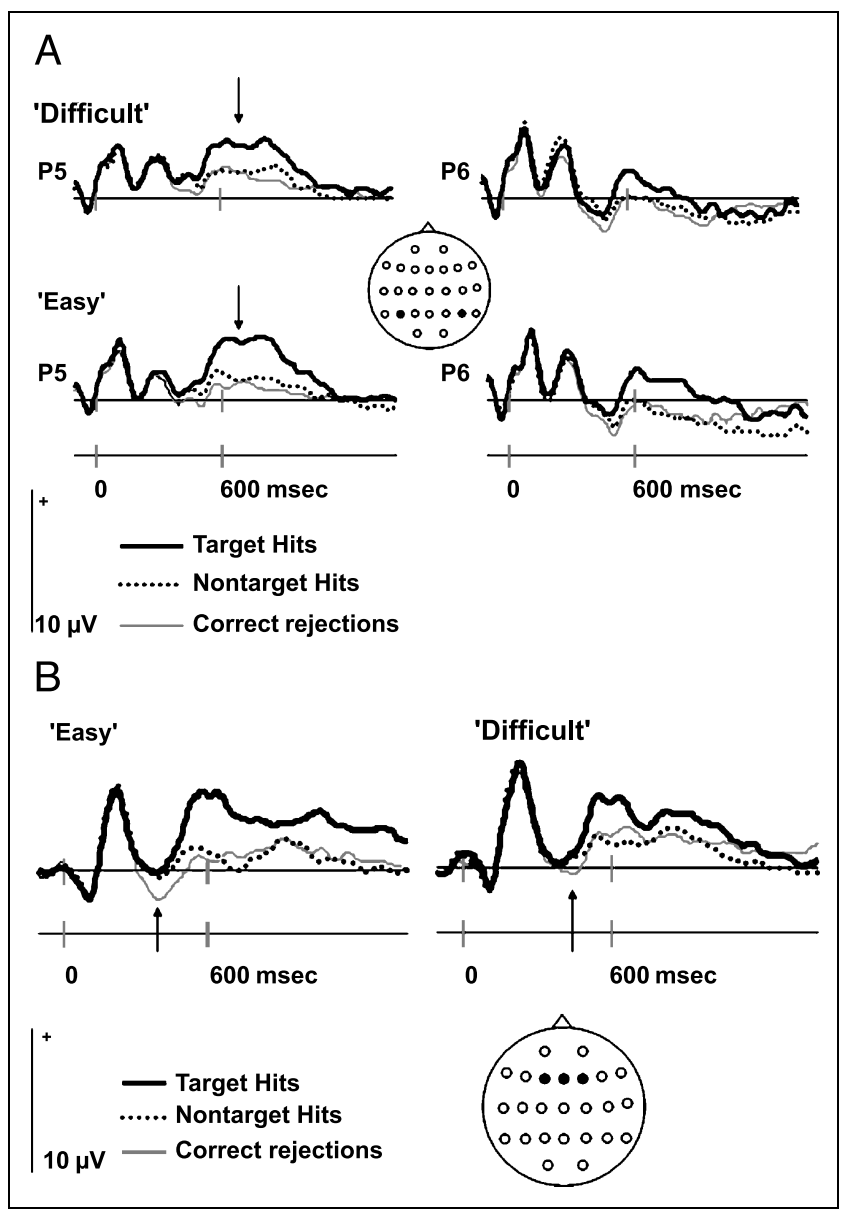

Figure 1. Averaged ERPs elicited by correctly classified targets, nontargets, and new items in both the difficult and easy conditions at (A) left and right parietal (P5/P6) electrode locations and (B) collapsed across the three midfrontal $(\mathrm{F} 3 / \mathrm{Fz} / \mathrm{F} 4)$ electrode locations. 


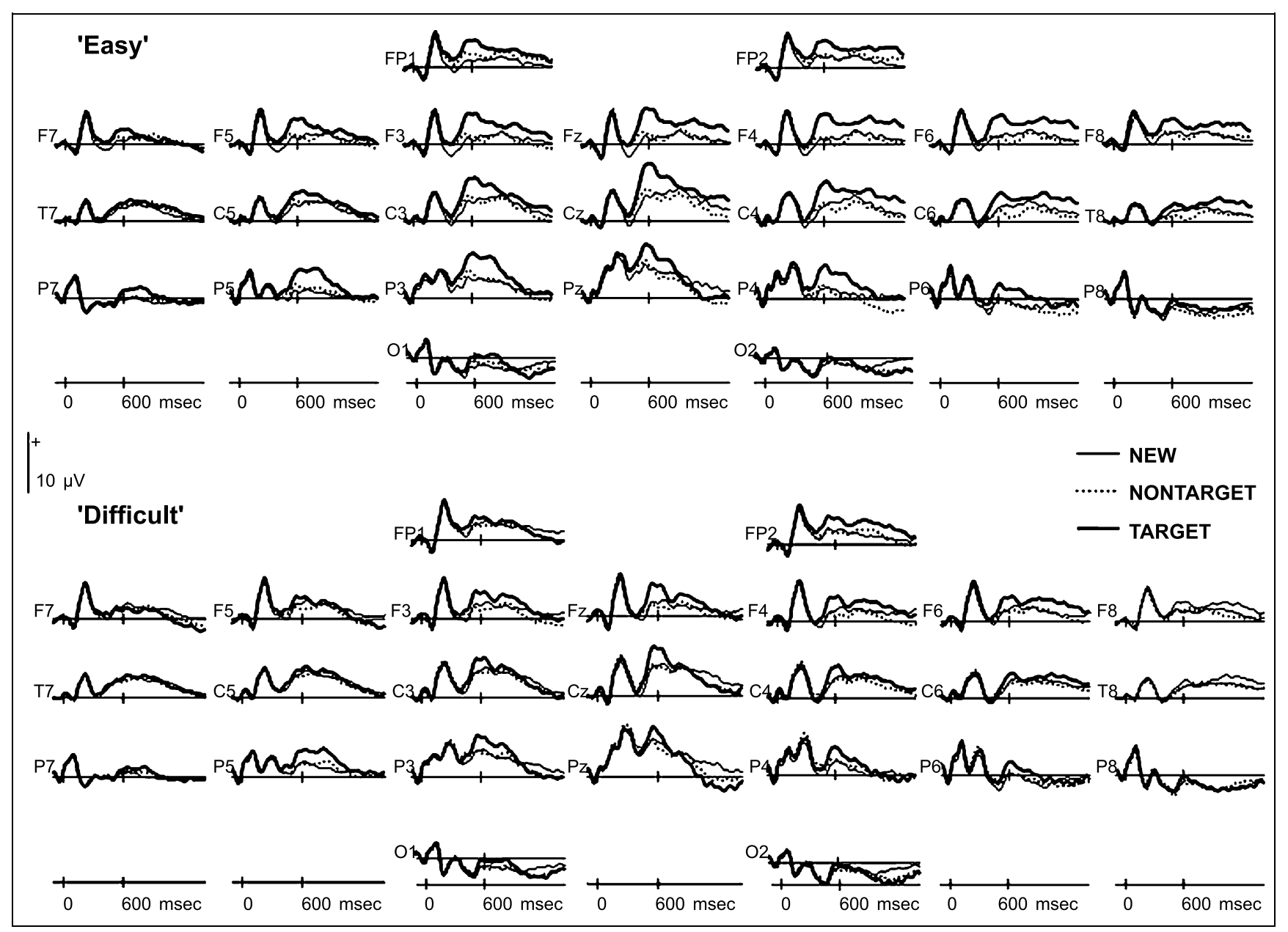

Figure 2. Averaged ERPs elicited by correctly classified targets, nontargets, and new items at all electrode sites in both the difficult and easy conditions.

gory are reported, and these are followed up with subsidiary pairwise comparisons where appropriate.

\section{Left Parietal Old/New Effect}

The analysis strategy employed to examine the left parietal old/new effects follows that adopted by Herron and Rugg (2003a) in order to ensure comparability of results; the ERPs elicited by targets, nontargets, and new items were analyzed over the 500- to $800-\mathrm{msec}$ time period at the left parietal electrode site indicated in Figure 1A. The ANOVA revealed a significant effect of Response Category, $F(1.7,28.5)=25.88, p<.001$, and a Response Category $\times$ Difficulty interaction, $F(1.8,30.1)=$ $3.51, p<.05$. The target/new item comparison gave rise to a main effect of Response Category, $F(1,17)=$ $35.28, p<.001$, and an interaction between Response Category and Difficulty, $F(1,17)=10.64, p<.005$, indicating that robust left parietal old/new effects were elicited by targets in both the easy and the difficult conditions, $F(1,17)=47.69, p<.001$, and $F(1,17)=$ $18.77, p<.001$, respectively, and that the effect was of greater magnitude in the easy condition. No effect of Response Category was observed in the nontarget/new item comparison. A main effect of Response Category was observed in the target/ nontarget comparison $F(1,17)=27.55, p<.001$, reflecting greater positivity for targets (see Figure 1A).

The same pattern of results was obtained when analysis was restricted to the nine participants with the lowest levels of target accuracy (mean accuracy of .53 and .60 in the difficult and easy conditions, respectively). Target ERPs were reliably more positive-going than those evoked by nontargets and new items, $F(1,8)=$ $27.01, p<.001 ; F(1,8)=17.20, p<.005$, respectively, and the latter categories did not differ.

\section{Early Frontal Old/New Effect}

Target, nontarget, and new item ERPs were analyzed at the three midfrontal sites shown in Figure 1B between 300 and $500 \mathrm{msec}$, in keeping with previous approaches (e.g., Rugg et al., 2002). ANOVA revealed a main effect of Response Category, $F(1.9,32.5)=7.31, p<.005$. Both 
target/new item and nontarget/new item comparisons gave rise to main effects of Response Category, $F(1,17)=17.49, p<.001 ; F(1,17)=5.77, p<.05$. Neither effect interacted with Difficulty. Targets and nontargets did not differ. Significant and statistically equivalent early frontal effects were therefore elicited by all correctly classified old items.

\section{Right Frontal Old/New Effect}

In keeping with previous work, analyses of this effect were confined to three right frontal sites (F4/F6/F8) between 800 and 1400 msec (Rugg et al., 2000; Donaldson \& Rugg, 1998; Wilding \& Rugg, 1996). ANOVA revealed a main effect of Response Category, $F(1.7,28.7)=7.98$, $p<.005$. Pairwise comparisons confirmed that targets were significantly more positive-going than new items, $F(1,17)=6.60, p<.025$, and nontargets, $F(1,17)=$ $19.43, p<.001$. Neither effect interacted with Difficulty. Nontarget and new item ERPs did not differ.

\section{Late Posterior Negativity}

The negative-going old/new effects evident over parietal sites were analyzed at $\mathrm{Pz}$ between 800 and $1400 \mathrm{msec}$, because this region is typically the focus of the effect (e.g., Johansson \& Mecklinger, 2003; Nessler \& Mecklinger, 2003). ANOVA gave rise to main effects of Difficulty, $F(1,17)=5.26, p<.05$, and Response Category, $F(1.5,26.3)=5.60, p<.025$, and an interaction between these two factors, $F(1.5,25.5)=4.58, p<.05$. These findings reflected significant negative old/new effects for targets, $F(1,17)=8.02, p<.025$, and nontargets, $F(1,17)=8.56, p<.01$, in the difficult condition and for nontargets in the easy condition, $F(1,17)=$ $12.02, p<.005$. The negativity elicited by targets in the easy condition did not reach significance $(p=.174)$.

\section{Subtraction Analyses}

Although the early frontal and right frontal old/new effects did not interact with Difficulty, visual inspection of the data suggested that both old/new effects were markedly larger in the easy condition (see Figures 1B and 2). A second set of analyses was therefore conducted to compare directly the amplitudes of each old/ new effect between conditions with greater sensitivity; these analyses were performed on data formed by subtracting new item ERPs from the respective old item ERPs within the relevant latency regions. The 300- to 500-msec analysis included target-new and nontargetnew data from the three midfrontal sites from both conditions (a $2 \times 2 \times 3$ design incorporating the factors of Difficulty, Response Category, and Site). A borderline effect of Difficulty was observed, $F(1,17)=4.07, p=$ .060 , reflecting the tendency for the early frontal old/ new effect to be larger in the easy condition. No effect of
Difficulty was obtained in the analysis restricted to right frontal sites over the 800- to 1400-msec epoch.

\section{Topographic Analyses}

A final set of analyses explored whether the ERP old/new effects reported above differed qualitatively (i.e., may have been generated by activity in at least partially nonoverlapping brain regions). These analyses were conducted upon the subtraction data described above, which was rescaled using the max-min method to avoid confounding changes in amplitude with changes in the shape of scalp distributions (McCarthy \& Wood, 1985). These analyses included data from a grid of 18 sites selected to sample activity recorded from across the scalp, and which allowed any differences to be identified by region; these sites included anterior, temporal, and parietal sites from the left and right hemispheres (F3, F4, F5, F6, F7, F8, C3, C4, C5, C6, T7, T8, P3, P5, P4, P6, P7, $\mathrm{P} 8)$, and included factors of Hemisphere, Anterior/Temporal/ Parietal, and Site (inferior/midlateral/superior). The first analysis included target-new and nontargetnew data from both conditions between 300 and $500 \mathrm{msec}$ and gave rise to no significant effect either of Response Category or Difficulty, suggesting that these four early frontal old/new effects shared the same neural generators.

The second analysis included easy and difficult targetnew data from the 300- to 500-, 500- to 800-, and 800- to 1400-msec epochs (nontarget-new data were not included as nontarget old/new effects were not significant from 500 to $800 \mathrm{msec}$ ). A number of interactions involving Epoch indicated that the scalp distribution of the old/new effects differed qualitatively across time, Epoch $\times$ Difficulty $\times$ Site: $F(2.5,42.7)=4.26, p<.025$; Epoch $\times$ Anterior $/$ Temporal/Parietal $\times$ Site: $F(3.7,63.4)=$ $3.32, p<.025$; Epoch $\times$ Hemisphere $\times$ Site: $F(2.1,36.3)=$ $16.62, p<.001$. Subsidiary analysis of the 300- to 500 and 500- to 800-msec epochs gave rise to an Epoch $\times$ Hemisphere $\times$ Anterior/Temporal/Parietal interaction, $F(1.3,21.5)=4.74, p<.05$, reflecting the fact that target old/new effects were focused primarily over frontal scalp sites from 300 to $500 \mathrm{msec}$ before additional left parietal foci emerged between 500 and $800 \mathrm{msec}$ (see Figure 3). Subsidiary analysis of the 500- to 800and 800 - to $1400-\mathrm{msec}$ latency regions gave rise to Epoch $\times$ Hemisphere $\times$ Anterior/Temporal/Parietal $\times$ Site, $F(2.5,42.0)=3.61, p<.05$, and Epoch $\times$ Difficulty $\times$ Site $F(1.8,30.5)=5.06, p<.025$, interactions, indicating that the distribution of the target old/new effect differed qualitatively across epoch-becoming focused over right frontal sites between 800 and 1400 msec with additional negative-going foci over midparietal sites (see Figure 3) - and that it was also modified by Difficulty. Further pairwise comparisons indicated that whereas easy and difficult target old/new effects did not differ during the 500- to 800-msec epoch, a Difficulty $\times$ 
Figure 3. Topographic maps showing the scalp distributions of target and nontarget old/new effects (collapsed across Difficulty) within the 300- to 500-msec epoch, and of the easy and difficult target old/new effects within the 500- to 800- and 800- to $1400-m s e c$ epochs. The maps were computed from the difference scores obtained by subtracting the ERPs evoked in one condition from those in another, as indicated above each map. The scales to the right of each map depict the voltage ranges (microvolts) of the differences between conditions.


'Easy' Target-New: 500-800 msec
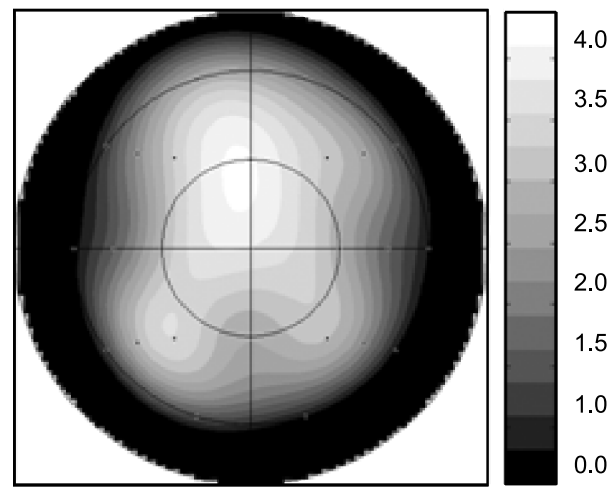

'Difficult' Target-New: 500-800 msec

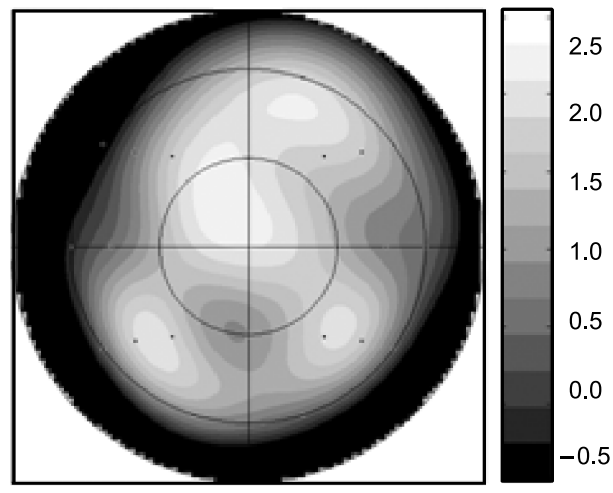

'Easy' Target-New: 800-1400 msec
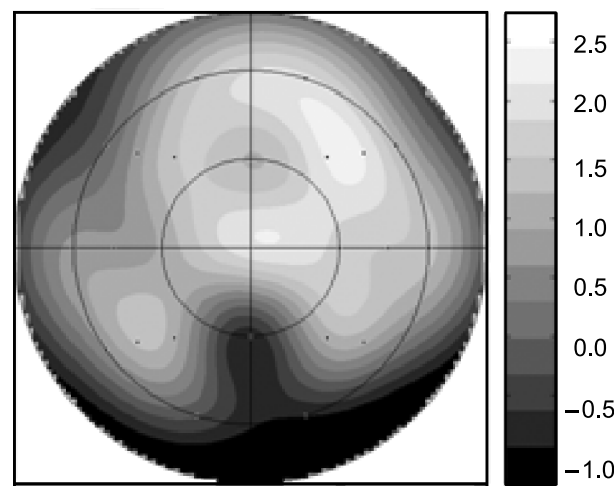

'Difficult' Target-New: 800-1400 msec

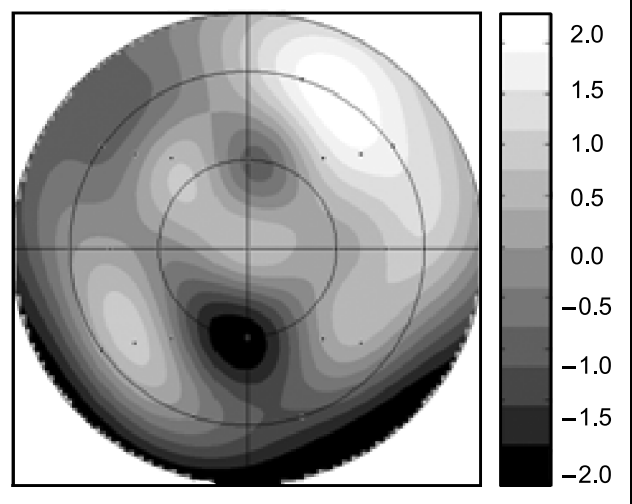

Site interaction, $F(1.9,32.0)=3.66, p<.05$, indicated that the distribution of the target old/new effect differed according to Difficulty in the 800- to 1400-msec epoch. Figure 3 suggests that the focus of the difficult target old/new effect extends across right inferior frontopolar sites whereas the easy target old/new effect is focused primarily over midlateral and superior right frontal sites.

\section{DISCUSSION}

As intended, the 40-min interval employed between the two study phases in the difficult condition decreased target accuracy relative to the easy condition, and to a level comparable with that reported by Herron \& Rugg (2003a) when targets were encoded in a shallow task. The difficult/easy manipulation, however, had no effect either on nontarget accuracy, new item accuracy, or on the RTs associated with correct responses to any of the three item types. Although the discrepancy in target accuracy between the difficult and easy conditions was not as large here (.07) as that reported by Herron and Rugg (.13) (possibly because participants were slightly fatigued during the easy condition as this was always performed last), the critical comparison-target accuracy in Herron and Rugg's shallow condition, and target 
accuracy in our difficult condition-showed that these indices were highly similar ( 0.63 and 0.65 , respectively). Moreover, although target/new discrimination was slightly higher in our difficult condition than in Herron and Rugg's shallow condition (.60 vs. .52), target/nontarget discrimination (computed by subtracting the likelihood of a target judgment to a nontarget from the likelihood of a correct target judgment) was almost identical in the two cases (.48 vs. .49). Therefore, a left parietal old/new effect should be observed for nontargets in our difficult condition if the hypothesis that low target accuracy discourages participants from adopting a target-specific retrieval strategy is correct.

Whereas robust left parietal old/new effects were observed for targets in both conditions, however, no such effect was detected for nontargets in either, although the animacy task in which nontargets were encoded has given rise to high levels of memory accuracy and reliable left parietal old/new effects in recognition memory tasks in previous studies (e.g., Herron, Quayle, \& Rugg, 2003; Duzel, Vargha-Khadem, Heinze, \& Mishkin, 2001; Otten \& Rugg, 2001). As noted in the Results section, furthermore, this pattern of parietal old/ new effects was unchanged even when the nine participants with the lowest target-accuracy scores were analyzed separately. Similarly, right frontal old/new effects were also selectively elicited by targets alone, an indication that participants engaged in the evaluation of retrieved information associated with targets but not nontargets. Participants therefore appeared to adopt a target-specific retrieval strategy in this experiment even when target accuracy was low, apparently suppressing or inhibiting the recollection of nontarget information. The present findings suggest that contrary to earlier hypotheses, the level of target accuracy is not the sole determinant of the conditions under which strategic retrieval will occur. Taken together with the findings of Herron and Rugg (2003a), it rather appears that targetspecific retrieval strategies are facilitated when the target-encoding task is sufficiently elaborative (or when the target/nontarget distinction criterion emphasizes the retrieval of elaboratively encoded information), even when target accuracy is low.

Our extension of Herron and Rugg's (2003a) findings itself raises two further possibilities; the important factor here may either be that targets themselves must be encoded elaboratively in order to facilitate target-specific retrieval strategies, or the critical factor may instead be that the contexts associated with targets and nontargets must be sufficiently distinct (as encoding targets and nontargets in different elaborative tasks would arguably render them more distinct than encoding one or both classes of items in a shallow encoding task). It should be noted that the sustained temporal segregation of targets and nontargets in the difficult condition may also have served to increase target/nontarget distinctiveness relative to Herron and Rugg's shallow condition. Although it appears that temporal segregation is not required for participants to adopt a target-specific retrieval strategy (as Herron \& Rugg, 2003b, reported ERP evidence of this even when targets and nontargets were intermixed within a single study phase), this does not rule out the possibility that temporal segregation of the two study contexts may serve as a contributing factor to the global level of target/nontarget distinctiveness. It is possible that strategic retrieval is facilitated when target/nontarget distinctiveness surpasses a certain criterion and that this level of distinctiveness may be created by a combination of factors such as encoding task, temporal segregation, and response requirements.

A second issue raised by the temporal segregation manipulation employed in the difficult condition is that this may have encouraged participants to employ recency information to distinguish between targets and nontargets as opposed to encoding operations. It is unlikely, however, that this can account for our findings, as the same pattern of old/new effects characteristic of strategic retrieval have been observed both when targets have been studied most recently (Herron \& Rugg, 2003a) and when targets and nontargets have been intermixed within a single study phase (Herron \& Rugg, 2003b). Yet even if participants did employ recency information when identifying targets in this study, some form of inhibition with regard to the retrieval of nontarget information must still have been exercised, as correct source judgments made on the basis of recency information also elicit reliable left parietal old/new effects (Tendolkar \& Rugg, 1998).

It is noteworthy that although the pattern of right frontal old/new effects followed that of the left parietal old/new effect (i.e., elicited by both classes of targets but neither class of nontargets), the distribution of the frontal effects varied with Difficulty. Figure 3 indicates that the effect was more diffuse and focused slightly more anteriorly in the difficult than in the easy condition. One way to explain these findings is via recourse to recent event-related fMRI studies of episodic retrieval, which have provided evidence that distinct right prefrontal regions play different roles in episodic retrieval (see Rugg \& Henson, 2003; Fletcher \& Henson, 2001, for reviews). In particular, whereas anterior portions of the right prefrontal cortex are associated with the amount of episodic information retrieved, the right dorsolateral prefrontal cortex is associated with retrieval-monitoring processes or effort (Henson, Rugg, Shallice, \& Dolan, 2000). It is therefore possible that distinct right prefrontal regions were differentially activated in our two conditions, with regions involved in the recovery of episodic information active in both conditions and regions involved in effortful retrieval activated to a greater extent in the difficult condition.

In addition to clarifying the conditions under which strategic retrieval is facilitated, the present findings also speak to an issue that has not yet been addressed in 
studies of strategic retrieval, but which is important for understanding the cognitive mechanisms underlying strategic retrieval processes, namely, the processing stage at which target-specific retrieval strategies operate. In this regard, it is important that the early frontal old/ new effect was evident and (at least statistically) of equal magnitude for both classes of old items. According to what is perhaps the prevailing view, this early frontal effect indexes familiarity (see Introduction), and if this account is correct, then the data support the view that strategic retrieval processes have little or no impact on the availability of familiarity for task judgments. The challenge for a familiarity account of the present data, however, stems from the fact that the target early frontal effect in the difficult condition was statistically equivalent to those elicited by the other three classes of old items. To the extent that familiarity decreases as the study-test interval increases (Yonelinas, 2002), then support for the familiarity account of the early frontal effect would have stemmed from a smaller target effect in the difficult condition compared to the effect elicited by nontargets in the difficult condition as well as by targets and nontargets in the easy condition (for an attenuation in midfrontal old/new effects according to lag, see Nessler \& Mecklinger, 2003). The fact that the amplitude of this effect was not significantly reduced can be seen to lend support to alternative accounts of the functional significance of the early frontal old/new effect, such as the view that the effect in fact indexes verbally mediated conceptual priming (Yovel \& Paller, 2004). Both of these accounts of the data, however, are constrained by the fact that the trend towards a Difficulty effect appears to be caused primarily by greater negativity for new items in the easy compared to the difficult condition (for related comments, see Tsivilis et al., 2001). For present purposes, the central point is that because the early frontal effect did not differ according to target/nontarget status, then whatever processes are indexed by this effect were influenced little by the strategic retrieval operations that influenced the recollection of nontarget information.

If the midfrontal effect, moreover, is an index of processes that can be diagnostic for old/new memory judgments, then one possibility is that in the tasks described here, participants engaged target-specific retrieval operations only once an item was identified as old. This account, however, runs counter to evidence that target-specific retrieval orientations influence neural activity associated with new items as well as old; ERPs elicited by new items differ depending on whether the sought-for information was studied in the form of words or pictures (Robb \& Rugg, 2002), a finding that was replicated using the exclusion task by Herron and Rugg (2003b). It therefore appears to be the case that targetspecific retrieval strategies are maintained throughout the entirety of a memory test, but that these strategies do not preclude recognition of nontargets on the basis of familiarity. According to this account, strategic retrieval processing is a consequence of the adoption of a preparatory state (a retrieval orientation: Rugg \& Wilding, 2000) that biases the way in which a retrieval cue is processed as a memory probe. This account is consistent with Herron and Rugg's (2003a) assertion that the mechanism underpinning strategic retrieval is "cue bias," a concept described by Anderson and Bjork (1994) as the way in which selection of specific "units" or aspects of the retrieval cue influences retrieval success; if inappropriate cues are used, then retrieval failure will occur (Anderson \& Bjork, 1994). More specifically, Anderson and Bjork describe a particular model of cue bias-"context bias"- -which is particularly relevant to this discussion; according to this model, retrieval failure will occur if the contextual representation used to guide the memory search does not match that which was present at encoding.

Taking the present findings and those of Herron and Rugg (2003a) together, it appears that participants may only be able to employ such biasing operations either when targets have been encoded elaboratively at study or when the target context is sufficiently distinct from the nontarget context. One possibility is that participants may prepare to retrieve or recapitulate the information that they generated internally in association with the external cue during the target study phase (i.e., maximizing the cue's compatibility with operations performed at study, in accordance with the transfer appropriate processing principle; Blaxton, 1989), and that such a retrieval strategy is possible only if a substantial amount of such information is available, for example, if participants have manipulated targets in a sufficiently elaborative task at study. It is notable that ERP exclusion tasks that have reported nontarget left parietal old/new effects have employed a target/nontarget exclusion criterion that emphasized attributes intrinsic to the stimulus, for example, voice (Wilding \& Rugg, 1996) and color (Cycowicz et al., 2001). Such information is not internally generated, would arguably not be unique to targets, and may be retrieved relatively more automatically together with the stimulus itself both for targets and for nontargets. An interesting question for future research will be to see if it is possible to elicit ERP evidence of target-specific strategic retrieval of information intrinsic to the stimulus.

The final ERP old/new effect reported above was the late posterior negativity (LPN), which occurred between 800 and $1400 \mathrm{msec}$. This negative-going old/new effect was evident over midparietal sites for all nontargets and for targets in the difficult condition. Although this effect has been reported in a number of ERP studies requiring the retrieval of contextual information (e.g., Cycowicz et al., 2001; Wilding \& Rugg, 1997), the functional significance of this effect remains unclear. In an important recent review and reanalysis, Johansson and Mecklinger (2003) decomposed the LPN into two com- 
ponents that were associated with the internal representation of the retrieved context and processes related to action monitoring (induced by response conflict), respectively. The latter class of processes have been linked to activations in the anterior cingulate cortex related to action monitoring (Mecklinger, 2000). Visual inspection of our data suggested that targets and nontargets in both conditions elicited an LPN, and indeed this effect reached significance for all old items with the exception of targets in the easy condition. However, easy targets also elicited a substantial right frontal effect of opposite polarity within the same latency region, and this may have attenuated the amplitude of the LPN. As nontargets failed to elicit the left parietal effect indicative of recollection, it is unlikely that the LPNs elicited by these items reflected a representation of retrieved contextual information. It therefore appears to be more likely that the LPNs observed here reflect primarily the second class of process described by Johansson and Mecklinger (2003); action-monitoring processes brought into operation by the response conflict induced by the fact that the different classes of old items required different responses.

A final question to be considered is why should participants rely exclusively on the retrieval of target information in the difficult condition when memory for these items was relatively poor? In reporting their findings, Herron and Rugg (2003a) argue that when target accuracy is high, the strategy adopted by participants involves probing each item for the presence or absence of contextual information from the target-encoding phase; items that do not elicit information diagnostic of the target source are excluded without any attempt to retrieve nontarget information. Our findings suggest that this strategy is not necessarily abandoned when target accuracy decreases, as long as targets have been manipulated in a sufficiently elaborative task. This is still a good strategy to adopt even if target accuracy is relatively low for the following reason: As the response demands emphasize the retrieval of target information while nontargets and new items share the alternate response, then the retrieval of nontarget information is arguably redundant because this will not necessarily increase the likelihood of responding accurately. Indeed, nontargets will be correctly classified if they are forgotten entirely. In order to maximize target accuracy, the best strategy to adopt will always be that which maximizes the overlap between the contextual specifications employed in the memory search and those defining the target study phase. Whether participants are able to do this, however, may depend on whether target/nontarget-encoding conditions are sufficiently elaborative and distinct to permit a target-specific, diagnostic memory search at test.

In summary, these results replicate partially, clarify, and extend the pattern of ERP old/new effects recently identified as being characteristic of "strategic recollec- tion," namely, the observation of robust left parietal old/ new effects for targets in the absence of nontarget left parietal old/new effects. The present findings clarify the experimental conditions that facilitate strategic retrieval, indicating that the nature of the encoding operations carried out at study influences the retrieval strategy adopted at test to a greater extent than does the level of target accuracy. Our findings also provide greater insight into the retrieval-processing stages at which strategic retrieval impacts, providing evidence that these strategic retrieval operations exert their influence selectively on processes tied closely to recollection.

\section{METHODS}

\section{Participants}

All participants were right-handed native English speakers, aged between 18 and 30. They were paid at a rate of $£ 5$ per hour and gave informed consent prior to the study. Data from three participants were discarded because there were fewer than 16 trials in one or more of the critical conditions (see below). Of the 18 participants contributing data towards analysis, 14 were women.

\section{Stimuli}

The stimuli comprised 480 low-frequency words (MRC psycholinguistic database: frequency 1-9 per million, Coltheart, 1981). Each experimental list was composed of two Study 1-Study 2 test blocks, each of which contained 240 words. Within each cycle, Study 1 and Study 2 phases each contained 80 words. All 160 words were repeated at test together with 80 new (unstudied) words in a randomized order. No words were repeated across blocks. Six experimental lists containing all 480 words were constructed, each of which contained six shorter word lists of 80 words each. Word lists were rotated across experimental lists, so that each word list served as targets, nontargets, and new items in each of the two conditions an equal number of times. Each experimental list was presented to three participants, and words in both test phases were presented in different randomized order for every participant.

\section{Procedure}

All stimuli were presented visually in white letters on a black background, on a monitor $1.2 \mathrm{~m}$ from the participant, and subtended maximum visual angles of $0.5^{\circ}$ (vertical) and $2.2^{\circ}$ (horizontal). Each participant was presented with two Study 1/Study 2/test cycles (Study 1 being the target study phase and Study 2 being the nontarget study phase). 


\section{Target Study Phase}

Each trial commenced with the presentation of a fixation point (an asterisk) for $100 \mathrm{msec}$. The screen was then blanked for 122 msec before the word was presented for 300 msec. The screen was then blanked until a response was made. Participants rated each item for pleasantness verbally on a 5-point scale from -2 (very unpleasant) to +2 (very pleasant). The first block incorporated a 40-min interval after the target study phase, during which time the electrode cap was applied to the participant. No such interval was employed in the second block.

\section{Nontarget Study Phase}

The structure of each trial was identical to the target study trials, with the exception that the screen was blanked for 1500 msec after the presentation of the study word. Participants responded on one key if the item was an animate object and on another if it was an inanimate object. This task is known to facilitate high levels of memory accuracy (Craik \& Lockhart, 1972) and robust left parietal old/new effects (Herron et al., 2003; Duzel et al., 2001; Otten \& Rugg, 2001).

\section{Test Phase}

Each trial began with the presentation of an asterisk for $100 \mathrm{msec}$, after which the screen was blanked for $122 \mathrm{msec}$. The test item was then presented for $300 \mathrm{msec}$, after which the screen was blanked for 2800 msec while the participant's response was recorded. Participants were instructed to respond on one key to items they rated for pleasantness (i.e., targets), and on another key both to new items and to items presented in the animacy task (i.e., nontargets). Participants were encouraged to balance speed and accuracy equally and to fixate centrally throughout. The keys designated for each response type were counterbalanced across participants. Responses quicker than 300 msec were treated as errors.

\section{ERP Recording and Analysis}

EEG was recorded from 25 silver/silver chloride electrodes at midline $(\mathrm{Fz}, \mathrm{Cz}, \mathrm{Pz})$ and left/right-hemisphere locations (FP1/FP2, F7/F8, F5/F6, F3/F4, T7/T8, C5/C6, C3/C4, P7/P8, P5/P6, P3/P4, O1/O2). Additional electrodes were placed on the mastoid processes. EOG was recorded from above and below the left eye (VEOG) and from the outer canthi (HEOG). EEG (0.03-40 Hz; 6 msec per point) was acquired referenced to $\mathrm{Fz}$ and rereferenced off-line to linked mastoids. Trials containing large EOG artifact were rejected, as were trials containing $\mathrm{A} / \mathrm{D}$ saturation or baseline drift exceeding $\pm 80 \mu \mathrm{V}$. Other EOG blink artifacts were corrected using a linear regres- sion estimate (Semlitsch, Anderer, Schuster, \& Presslich, 1986).

\section{Acknowledgments}

This research was supported by the Wellcome Trust.

Reprint requests should be sent to J. E. Herron, School of Psychology, Cardiff University, Cardiff CF10 3YG, Wales, UK, or via e-mail: herronje@cardiff.ac.uk.

\section{REFERENCES}

Anderson, M. C., \& Bjork, R. A. (1994). Mechanisms of inhibition in long term memory. In D. Dagenbach \& T. H. Carr (Eds.), Inbibitory processes in attention, memory, and language (pp. 265-325). San Diego, CA: Academic Press.

Blaxton, T. (1989). Investigating dissociations among memory measures: Support for a transfer-appropriate processing framework. Journal of Experimental Psychology: Learning, Memory, and Cognition, 15, 657-668.

Coltheart, M. (1981). The MRC psycholinguistic database. Quarterly Journal of Experimental Psychology A, 33, 497-505.

Craik, F. I. M., \& Lockhart, R. S. (1972). Levels of processing: A framework for memory research. Journal of Verbal Learning and Verbal Behaviour, 16, 519-533.

Curran, T. (2000). Brain potentials of recollection and familiarity. Memory and Cognition, 28, 923-938.

Curran, T., Schacter, D. L., Johnson, M., \& Spinks, R. (2001). Brain potentials reflect behavioral differences in true and false recognition. Journal of Cognitive Neuroscience, 13, 201-216.

Cycowicz, Y. M., Friedman, D., \& Snodgrass, J. G. (2001). Remembering the colour of objects: An ERP investigation of source memory. Cerebral Cortex, 11, 322-334.

Donaldson, D. I., \& Rugg, M. D. (1998). Recognition memory for new associations: Electrophysiological evidence for the role of recollection. Neuropsychologia, 36, 377-395.

Duzel, E., Vargha-Khadem, F., Heinze, H. J., \& Mishkin, M. (2001). Brain activity evidence for recognition without recollection after early hippocampal damage. Proceedings of the National Academy of Sciences, U.S.A., 98, 8101-8106.

Duzel, E., Yonelinas, A. P., Mangun, G. R., Heinze, H. J., \& Tulving, E. (1997). Event-related brain potential correlates of two states of conscious awareness in memory. Proceedings of the National Academy of Sciences, U.S.A., 94, 5973-5978.

Dywan, J., Segalowitz, S. J., \& Arsenault, A. (2002).

Electrophysiological response during source memory decisions in older and younger adults. Brain and Cognition, 49, 322-340.

Dywan, J., Segalowitz, S. J., \& Webster, L. (1998). Source monitoring: ERP evidence for greater reactivity to non-target information in older adults. Brain and Cognition, 36, $390-430$.

Dywan, J., Segalowitz, S. J., Webster, L., Hendry, K., \& Harding, J. (2001). Event-related potential evidence for age-related differences in attentional allocation during a source monitoring task. Developmental Neuropsychology, 19, 99-120.

Fletcher, P. C., \& Henson, R. N. A. (2001). Frontal lobes and human memory: Insights from functional neuroimaging. Brain, 124, 849-881.

Friedman, D., \& Johnson, R. (2000). Event-related potential (ERP) studies of memory encoding and retrieval: A selective review. Microscopy Research and Techniques, 51, 6-28.

Greenhouse, G. W., \& Geisser, S. (1959). On methods in the 
analysis of repeated measures designs. Psychometrika, 49, 95-112.

Henson, R. N. A., Rugg, M., Shallice, T., \& Dolan, R. J. (2000). Confidence in recognition memory for words: Dissociating right prefrontal roles in episodic retrieval. Journal of Cognitive Neuroscience, 12, 913-923.

Herron, J. E., \& Rugg, M. D. (2003a). Strategic influences on recollection in the exclusion task: Electrophysiological evidence. Psychonomic Bulletin and Review, 10, 703-710.

Herron, J. E., \& Rugg, M. D. (2003b). Retrieval orientation and the control of recollection. Journal of Cognitive Neuroscience, 15, 843-854.

Herron, J. E., Quayle, A. H., \& Rugg, M. D. (2003). Probability effects on event-related potential correlates of recognition memory. Cognitive Brain Research, 16, 66-73.

Jacoby, L. L. (1991). A process dissociation framework: Separating automatic from intentional uses of memory. Journal of Memory and Language, 30, 513-541.

Jacoby, L. L. (1998). Invariance in automatic influences of memory: Toward a user's guide for the process-dissociation procedure. Journal of Experimental Psychology: Learning, Memory, and Cognition, 24, 3-26.

Johansson, M., \& Mecklinger, A. (2003). The late posterior negativity in ERP studies of episodic memory: Action monitoring and retrieval of attribute conjunctions. Biological Psychology, 64, 91-117.

McCarthy, G., \& Wood, C. C. (1985). Scalp distributions of event-related potentials: An ambiguity associated with analysis of variance models. Electroencephalography and Clinical Neurophysiology, 62, 203-208.

Mecklinger, A. (2000). Interfacing mind and brain: A neurocognitive model of recognition memory. Psychophysiology, 37, 565-582.

Nessler, D., \& Mecklinger, A. (2003). ERP correlates of true and false recognition after different retention delays: Stimulus- and response-related processes. Psychophysiology, 40, 146-159.

Otten, L. J., \& Rugg, M. D. (2001). Electrophysiological correlates of memory encoding are task-dependent. Cognitive Brain Research, 12, 11-18.

Robb, W. G. K., \& Rugg, M. D. (2002). Electrophysiological dissociation of retrieval orientation and retrieval effort. Psychonomic Bulletin and Review, 9, 583-589.

Rugg, M. D., \& Allan, K. (2000). Event-related potential studies of memory. In E. Tulving \& F. I. M. Craik (Eds.), The Oxford handbook of memory (pp. 521-537). Oxford: Oxford University Press.

Rugg, M. D., Allan, K., \& Birch, C. S. (2000). Electrophysiological evidence for the modulation of retrieval orientation by depth of study processing. Journal of Cognitive Neuroscience, 12, 664-678.

Rugg, M. D., \& Henson, R. N. A. (2003). Episodic memory retrieval: An (event-related) functional neuroimaging perspective. In A. E. Parker, E. L. Wilding, \& T. J. Bussey (Eds.), The cognitive neuroscience of memory: Episodic encoding and retrieval (pp. 5-38). Hove, UK: Psychology Press.

Rugg, M. D., Herron, J. E., \& Morcom, A. M. (2002). Electrophysiological studies of retrieval processing In L. R. Squire \& D. L. Schacter (Eds.), Neuropsychology of memory (pp. 154-165). New York: Guilford Press.

Rugg, M. D., Mark, R. E., Walla, P., Schloerscheidt, A., Birch, C. S., \& Allan, K. (1998). Dissociation of the neural correlates of implicit and explicit memory. Nature, 392, 595-598.

Rugg, M. D., \& Wilding, E. L. (2000). Retrieval processing and episodic memory. Trends in Cognitive Sciences, 4, 108-115.

Semlitsch, H. V., Anderer, P., Schuster, P., \& Presslich, O. (1986). A solution for reliable and valid reduction of ocular artifacts, applied to the P300 ERP. Psychophysiology, 23, 695-703.

Smith, M. E. (1993). Neurophysiological manifestations of recollective experience during recognition memory judgements. Journal of Cognitive Neuroscience, 5, 1-13.

Spencer, K., Abad, E. V., \& Donchin, E. (2000). On the search for the neurophysiological manifestation of recollective experience. Psychophysiology, 37, 494-506.

Tendolkar, I., \& Rugg, M. D. (1998). Electrophysiological dissociation of recency and recognition memory. Neuropsychologia, 36, 477-490.

Tsivilis, D., Otten, L. J., \& Rugg, M. D. (2001). Context effects on the neural correlates of recognition memory: an electrophysiological study. Neuron, 31, 497-505.

Ullsperger, M., Mecklinger, A., \& Muller, U. (2000). An electrophysiological test of directed forgetting: The role of retrieval inhibition. Journal of Cognitive Neuroscience, 12, 924-940.

Wilding, E. L. (2000). In what way does the parietal ERP $\mathrm{old} / \mathrm{new}$ effect index recollection? International Journal of Psychophysiology, 35, 81-87.

Wilding, E. L., \& Rugg, M. D. (1996). An event-related potential study of recognition memory with and without retrieval of source. Brain, 119, 889-905.

Wilding, E. L., \& Rugg, M. D. (1997). Event-related potentials and the recognition memory exclusion task. Neuropsychologia, 35, 119-128.

Wilding, E. L., \& Sharpe, H. L. (2004). The influence of response-time demands on electrophysiological correlates of successful episodic retrieval. Cognitive Brain Research, $18,185-195$.

Yonelinas, A. P. (2002). The nature of recollection and familiarity: A review of 30 years of research. Journal of Memory and Language, 46, 441-517.

Yovel, G., \& Paller, K. A. (2004). The neural basis of the butcher-on-the-bus phenomenon: When a face seems familiar but is not remembered. Neuroimage, 21, 789-800. 